\title{
Placental Growth Factor, a Member of the VEGF Family, Contributes to the Development of Choroidal Neovascularization
}

Jean-Marie Rakic, ${ }^{1}$ Vincent Lambert, ${ }^{2}$ Laetitia Devy, ${ }^{2}$ Aernout Luttun, ${ }^{3}$ Peter Carmeliet, ${ }^{3}$ Caret Claes, ${ }^{4}$ Laurent Nguyen, ${ }^{5}$ Jean-Michel Foidart, ${ }^{2}$ Agnès Noël, ${ }^{2}$ and Carine Munaut ${ }^{2}$

From the ${ }^{1}$ Department of Ophthalmology, University Hospital. Sart-Tilman, Liège, Belgium; the ${ }^{2}$ Laboratory of Tumor and Development Biology and the ${ }^{5}$ Center for Cellular and Molecular Neurobiology, University of Liège, Liège, Belgium; the ${ }^{3}$ Center for Transgene Technology and Gene Therapy, Katholieke Universiteit Leuven, Leuven, Belgium; and the ${ }^{4}$ Department of Ophthalmology, Middelheim Hospital, Antwerpen, Belgium.

\begin{abstract}
PURPOSE. VEGF has been shown to be necessary, but not sufficient alone, for the development of subretinal pathologic angiogenesis. In the current study, the influence of placental growth factor (PlGF), a member of the VEGF family, in human and experimental choroidal neovascularization (CNV) was investigated.
\end{abstract}

METHODS. The presence of VEGF family member mRNA was evaluated by RT-PCR in neovascular membranes extracted during surgery. The spatial and temporal pattern of VEGF isoforms and PlGF mRNA expression were explored by using the laser capture catapulting technique and RT-PCR in a murine laser-induced model and in vitro. PlGF expression was also studied in human donor eyes. The influence of endogenous PlGF was evaluated in deficient mice $\left(\mathrm{PlGF}^{--}\right)$and by antibody-mediated neutralization of the PlGF receptor.

RESULTS. Human neovascular membranes consistently expressed VEGF-A, -B, and -C; PlGF; and VEGFR-1 and -2. The $\mathrm{VEGF}_{120}$ isoform mRNA was primarily induced in early stages of angiogenesis in vivo and in vitro. PlGF mRNA expression was present in the intact choroid and significantly upregulated during the course of experimental CNV. Both deficient PlGF expression in $\mathrm{PlGF}^{-/-}$mice and PlGF receptor neutralization in wild-type mice prevented the development of choroidal neovascularization induced by laser.

ConCLUSIONS. These observations demonstrate the participation of PlGF in experimental CNV. They identify therefore PlGF as an additional promising target for ocular antiangiogenic strategies.

Vascular endothelial growth factor type A (VEGF-A, commonly referred to as VEGF) has been implicated in several retinal disorders characterized by neovascularization, including the exudative form of age-related macular degeneration (AMD). ${ }^{1,2}$ Several reports have demonstrated the simultaneous presence of VEGF-A and its receptor VEGFR-2 (also known as Flk1) in neovascular membranes and in experimental choroidal neovascularization (CNV). ${ }^{3-5}$ These observations have lead to the development of several preclinical studies using anti-VEGF strategy, such as anti-VEGF antibody or aptamer. ${ }^{6,7}$

However, VEGF-A is expressed throughout the normal retina without the development of endothelial cell proliferation of vascular leakage ${ }^{8}$ suggesting that the VEGF system may be only one part of a complex network of pro- and antiangiogenic mechanisms operating both under physiologic and pathologic conditions. ${ }^{9}$ Furthermore, isolated overexpression of VEGF-A either in photoreceptors or in retinal pigment epithelium of transgenic mice does not result in the formation of $\mathrm{CNV},{ }^{10,11}$ unless some kind of local trauma (i.e., trauma associated with sustained release models or subretinal injection of viral vectors) is induced in Bruch's membrane. ${ }^{12,13}$ This indicates that the development of CNV necessitates either a combination of multiple proangiogenic growth factors in cooperation with VEGF-A, a simultaneous decrease in the expression of antiangiogenic agents (such as demonstrated in vitro for pigment epithelium-derived growth factor-PEDF), ${ }^{14,15}$ or that damage to Bruch's membrane and photoreceptors is a necessary condition.

The VEGF family, besides VEGF-A, includes also VEGF-B, -C, and -D and placental growth factor (PlGF), ${ }^{16}$ and the presence of these proteins has recently been reported in human CNV membranes. ${ }^{17}$

\footnotetext{
* Supported by grants from Les Amis des Aveugles, Ghlin, and the Funds for Medical Scientific Research. CM is a research associate and AN is a senior research associate from the National Fund for Scientific Research, Belgium.
} 
PlGF and its receptor Flt1 (also referred to as VEGFR-1) received little attention until a recent report demonstrating that although PlGF-deficient mice develop normally, loss of PlGF blocks pathologic angiogenesis and vascular leakage in cancer, ischemia, and wound healing. ${ }^{18}$ In the cornea pellet model, PlGF was as effective as VEGF-A in inducing angiogenesis. ${ }^{19}$ In the retina, PIGF expression was not upregulated by hypoxia, ${ }^{20}$ but PlGF deficiency reduced by $60 \%$ the amount of retinal neovascularization in the retinopathy of prematurity (ROP) model, ${ }^{18}$ and PIGF was proangiogenic on retinal endothelial cells. ${ }^{21}$ Finally, PlGF was detected in the vitreous and in the neovascular membranes in proliferative diabetic retinopathy. ${ }^{22}$

The potential involvement of PlGF has not been investigated in human or experimental CNV.

In the present study, in addition to VEGF-A, human choroidal neovascular membranes excised during surgery for macular translocation (end-stage disease) also expressed PIGF, VEGF-B and -C, and VEGFR-1 and -2 mRNAs. The spatial and temporal expression profiles of VEGF isoforms and of PlGF were further evaluated by RT-PCR in a murine laser-induced CNV model. To characterize the relative contribution of PlGF to the development of experimental choroidal angiogenesis, we induced subretinal neovascularization in PlGFdeficient mice $\left(\mathrm{PlGF}^{-/}\right)$, in wild-type (WT) control mice, and in mice treated with an inhibitor of PlGF receptor.

TABLE 1: RT-PCR Parameters

\begin{tabular}{|c|c|c|c|c|}
\hline $\begin{array}{l}\text { Gene and Accession } \\
\text { Number }\end{array}$ & Position & Oligonucleotide Sequence (5'- 3') & $\begin{array}{l}\text { Size of PCR } \\
\text { Product (bp) }\end{array}$ & $\begin{array}{l}\text { Number of } \\
\text { Cycles }\end{array}$ \\
\hline \multirow[t]{2}{*}{ 28S, U13369 } & $12403 \mathrm{~F}$ & GTTCACCCACTAATAGGGAACGTGA & 212 & 19 \\
\hline & 12614R & GGATTCTGACTTAGAGGCGTTCAGT & & \\
\hline \multirow[t]{3}{*}{ m,hVEGF-A, AH001553 } & $1208 \mathrm{~F}$ & CCTGGTGGACATCTTCCAGGAGTA & 407 & 33 \\
\hline & $1687 \mathrm{R}$ & CTCACCGCCTCGGCTTGTCACA & 347 & \\
\hline & & & 275 & \\
\hline \multirow[t]{2}{*}{ hVEGF-B, U52819 } & $98 \mathrm{~F}$ & CAGAGGAAAGTGGTGTCATGGA & 223 & 33 \\
\hline & 320R & ACCGGATCATGAGGATCTGCA & & \\
\hline \multirow[t]{2}{*}{ hVEGF-C, NM_005429 } & 947F & CTCTCAAGGCСССАААССА & 152 & 33 \\
\hline & 1098R & AGGTCTTGTTCGCTGCCTGA & & \\
\hline \multirow[t]{2}{*}{ hVEGF-D, NM_004469 } & $1146 \mathrm{~F}$ & GATCGCTGTTCCCATTCCA & 152 & 33 \\
\hline & 1297R & ATCATGTGTGGCCCACAGAGA & & \\
\hline \multirow[t]{2}{*}{ hVEGFRl, AF063657 } & $2438 \mathrm{~F}$ & TCCCTTATGATGCCAGCAAGT & 79 & 33 \\
\hline & 2516R & CСAAAAGCСССТСТTССАА & & \\
\hline \multirow[t]{2}{*}{ hVEGFR2, AF063658 } & $791 \mathrm{~F}$ & CTTCGAAGCATCAGCATAAGAAACT & 156 & 33 \\
\hline & 946R & TGGTCATCAGCCCACTGGAT & & \\
\hline \multirow[t]{2}{*}{ hPIGF, X54936 } & $583 \mathrm{~F}$ & GGCGATGAGAATCTGCACTGT & 164 & 33 \\
\hline & $746 \mathrm{R}$ & CACCTTTCCGGCTTCATCTTC & & \\
\hline \multirow[t]{2}{*}{ mPIGF, BCO16567 } & $624 \mathrm{~F}$ & AGATCTTGAAGATTCСССССА & 130 & $35^{*}$ \\
\hline & $753 \mathrm{~F}$ & TTCCССTTGGTTTTCСTCСTT & & \\
\hline
\end{tabular}

m, mouse; h, human. * 45 Cycles for LPC

\section{MATERIALS AND METHODS}

\section{RT-PCR Analysis of Neovascular Membranes}

Four consecutive submacular CNV specimens were completely removed during surgery for $360^{\circ}$ macular translocation performed on patients with exudative AMD, either not amenable to conventional laser/photodynamic therapy (presence of occult neovessels or submacular bleeding) or in one patient, owing to a severe recurrence a few months after successful medical treatment. The neovascular membranes were snap frozen in liquid nitrogen and stored at $-80^{\circ} \mathrm{C}$. The methods conformed to the provisions of the Declaration of Helsinki for research involving human subjects. Ten human donor eyes (from the Cornea Bank, Liège, Belgium) were used to evaluate the expression of PIGF in intact neural retina and RPE-choroid. 
$\mathrm{CNV}$ was induced in mice by multiple argon laser burns, as previously described. ${ }^{23}$ Animals were killed at days $3,5,10,14,20$, and 40 , and the eyes were enucleated. The posterior segments (neural retina and RPE-choroid complex) were dissected and immediately frozen in liquid nitrogen.

The frozen tissues were pulverized (Dismembrator; B. Braun Biotech International, GmbH, Melsungen, Germany), and total RNA was extracted with a kit (RNeasy; Qiagen, Paris, France), according to the manufacturer's protocol. 28S rRNA was amplified with an 10-ng aliquot of total RNA (for tissues) or $1 \mu \mathrm{L}$ of total RNA (laser pressure catapulting [LPC] samples) using an amplification kit (GeneAmp Thermostable rTth reverse transcriptase RNA PCR kit; Applied Biosystems, Foster City, CA) and two pairs of primers (Eurogentec, Liège, Belgium) with the oligonucleotide sequences shown in Table 1. For VEGF-A, a synthetic RNA (CTR1, internal control) giving rise to a 311-bp fragment was incorporated in the experiment involving aortic rings. Reverse transcription was performed at $70^{\circ} \mathrm{C}$ for 15 minutes followed by 2 minutes of incubation at $95^{\circ} \mathrm{C}$ for denaturation of RNA-DNA heteroduplexes. Amplification began with 15 seconds at $94^{\circ} \mathrm{C}, 20$ seconds at $60^{\circ} \mathrm{C}$, and 10 seconds at $72^{\circ} \mathrm{C}$. RT-PCR products were resolved on $2 \%$ agarose gels and analyzed with a fluorescence imager (Fluor-S Multi-Imager; Bio-Rad, Hertfordshire, UK) after staining with ethidium bromide (FMC BioProducts, Philadelphia, PA).

\section{Laser Pressure Catapulting}

To evaluate the spatial and temporal pattern of VEGF-A and PIGF expression in experimental CNV, eyes were enucleated at selected intervals (days 3, 5, and 14) after laser induction, embedded in OCT compound (Tissue Tek; Miles Laboratories, Naperville, IL), and frozen in liquid nitrogen. Serial frozen sections $(n=8-10)$ were mounted directly onto 1.35-mm thin polyethylene foil (Palm, Wolfratshausen, Germany). The system (RobotMicrobeam; Palm) focused the laser (337 nm) on the specimen enabling the catapulting of the entire selected area into the microfuge cap. The subretinal CNV area (see Fig. 2A, inside the dotted line) and an adjacent intact chorioretinal zone (control) were microdissected separately on frozen sections (10 $\mu \mathrm{m}$ thick). The specimens were covered with $100 \mu \mathrm{L}$ lysis buffer and total RNA isolation was performed with an RNA isolation kit (PUREscript; Biozym, Landgraaf, The Netherlands) according to the manufacturer's protocol. Total RNA was dissolved in a 10- $\mu$ L RNA hydration solution supplied by the manufacturer, and RT-PCR was performed as previously described. ${ }^{23}$

\section{Genetically Modified Mice}

We used the progeny of heterozygous breeding pairs of mice with targeted disruption of PlGF, as described. ${ }^{18}$ Brothers and sisters from the same litter were intercrossed by brother-sister mating to obtain homozygous offspring genotyped by RT-PCR. Two- to 5-month-old pigmented PlGF-deficient mice (PlGF ${ }^{-/}$) and the corresponding WT mice $\left(\mathrm{PlGF}^{+/+}\right)$of either sex were used in experiments in which neovascular membranes were quantified. The animals (five or more in each group) used in this study were maintained in a 12-hour light- dark cycle and had free access to food and water. All animal experiments were performed in compliance with the ARVO Statement for the Use of Animals in Ophthalmic and Vision Research.

\section{Murine Model of Laser-Induced CNV}

CNV was induced in mice by four burns (at the 6, 9, 12, and 3 o'clock positions around the optic disc) using a green argon laser (532 nm; 50 - $\mu \mathrm{m}$ diameter spot size; 0.05 -second duration; $400 \mathrm{~mW}$ ) as previously described. ${ }^{23,24}$ The eyes were enucleated at day 14, embedded in OCT compound (Tissue Tek; Miles Laboratories), and frozen in liquid nitrogen for cryostat sectioning. To quantify CNV, frozen serial sections were cut throughout the entire extent of each burn, and the thickest region (minimum of four per lesion) was used for the quantification. ${ }^{23,24}$ Using a computer-assisted image analysis system (Olympus Micro Image version 3.0 for Windows 95/NT; Olympus Optical Co. Europe, Hamburg, Germany), neovascularization was estimated by the ratio (B-C) of the thickness from the bottom of the pigmented choroidal layer to the top of the neovascular membrane (B), to the thickness of the intact-pigmented choroid adjacent to the lesion (C). 
FIGURE 1: VEGF family $m R N A$ expression in exudative AMD. Representative example of VEGF-A, -B, -C; PlGF; VEGFR-1 and -2 mRNA expression in surgically extracted choroidal neovascular membranes of four patients (subjects 1-4) with aggressive exudative AMD. Total RNA (approximately $10 \mathrm{ng}$ ) was subjected to RT-PCR. The $28 \mathrm{~S}$ rRNA was used to assess the total amount of RNA loaded. Apparent differences in the pattern of mRNA expression in the CNV of different patients were not quantified. M, molecular marker. Right: PCR products size (in base pairs; see also Table 1).

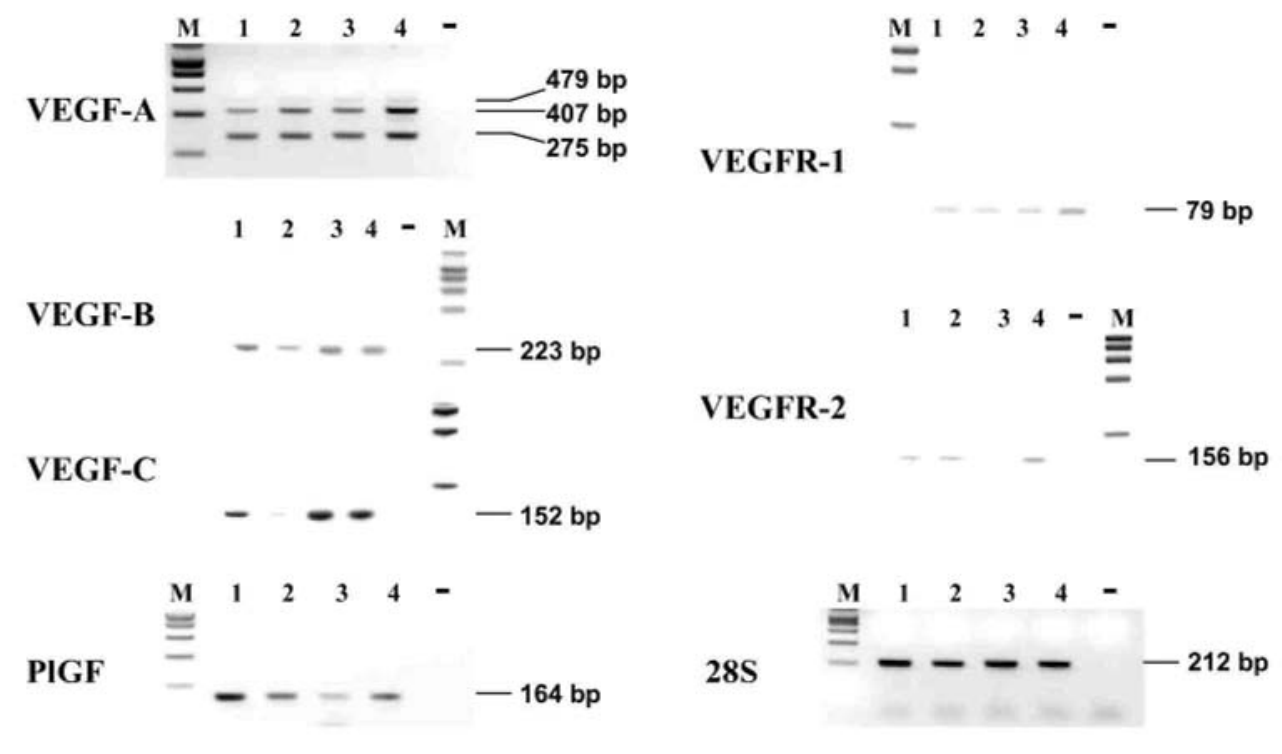

\section{Neutralization of PIGF Receptor}

To evaluate whether inhibition of the PIGF pathway would inhibit the development of laser-induced CNV, WT mice were intraperitoneally injected with $500 \mu \mathrm{g}$ monoclonal rat anti-mouse-Flt1 (clone MF-1, a generous gift of Daniel J. Hicklin, ImClone Systems Inc., New York, NY). Control mice were similarly injected three times per week with an equal dose of rat immunoglobulins (IgG; Sigma-Aldrich, Bornem, Belgium). ${ }^{25}$ At day 14, mice treated with anti-Flt1 and controls were anesthetized and intravenously perfused with $0.3 \mathrm{~mL}$ phosphate-buffered saline (PBS) containing $50 \mathrm{mg} / \mathrm{mL}$ fluorescein-labeled dextran (2 x 10 average molecular weight; SigmaAldrich), as previously described. ${ }^{26,27}$ The eyes were removed and either embedded in OCT compound (for B-C ratio evaluation on frozen serial sections) or dissected to obtain choroidal flatmounts embedded in aqueous mounting medium (Aquamount; Sigma-Aldrich). Flatmounts were examined by fluorescence microscope (Axiovert 135; Carl Zeiss Meditech, Zaventem, Belgium) and laser scanning confocal microscope (Bio-Rad), and images were digitized. Image-analysis software (Photoshop 5.0; Adobe Systems, Mountain View, CA) was used to measure the total area of hyperfluorescence associated with each laser impact.

\section{Aortic Ring Model}

Mice aortic expiant cultures were prepared as described ${ }^{28}$ Briefly, ring-shaped expiants of mouse aorta were embedded in a rat tail interstitial collagen (type I) gel (Collagen R; Serva, Heidelberg, Germany) and allowed to gel in cylindrical agarose wells. Aortic rings were maintained at $37^{\circ} \mathrm{C}$ in $6 \mathrm{~mL}$ of MCDB131 (Life Technologies, Paisley, Scotland) supplemented with $25 \mathrm{mM} \mathrm{NaHCO}_{3}, 2.5 \%$ mouse serum, $1 \%$ glutamine, $100 \mathrm{U} / \mathrm{mL}$ penicillin, and $100 \mathrm{\mu g} / \mathrm{mL}$ streptomycin. The endothelial origin of migrating cells was verified by Dil-Ac-LDL (SigmaAldrich) incorporation in the aorta ring before it was embedded in collagen gel $\left(10 \mu \mathrm{g} / \mathrm{mL}\right.$ for 4 hours at $\left.37^{\circ} \mathrm{C}\right)$. At day 7, aortic rings (see Fig. 2C, inside the dotted line) were removed from the collagen gel and frozen individually in liquid nitrogen. Migrating endothelial cells from three mice were removed by centrifugation after treatment of the collagen gel with $1 \mathrm{mg} / \mathrm{mL}$ collagenase A (Sigma) for 10 minutes at $37^{\circ} \mathrm{C}$, pooled, and stored in liquid nitrogen until RT-PCR.

\section{Immunohistochemistry}

Cryostat sections (5 $\mu$ m thick) were fixed in paraformaldehyde $1 \%$ in $0.07 \mathrm{M}$ phosphate-buffered saline (PBS; $\mathrm{pH}$ 7.0) for 5 minutes or in acetone for 10 minutes at room temperature and then incubated with the primary antibody. Antibodies raised against type IV collagen (guinea pig polyclonal, produced in our laboratory; diluted 1/100), mouse platelet- endothelial cell adhesion molecule (PECAM; rat monoclonal, PharMingen, San Diego, 
CA; diluted 1:20), VEGF (rabbit polyclonal, Santa Cruz Biotechnology, Santa Cruz, CA; diluted 1:100), and murine PIGF (rat monoclonal, R\&D Systems, Abingdon, UK; diluted 1:50) were incubated for 1 hour at room temperature. The sections were washed in PBS (three times for 10 minutes each), and appropriate secondary antibody conjugated to horseradish peroxidase (HRP), fluorescein-isothiocyanate (FITC), or tetramethylrhodamine isothiocyanate (TRITC) was added: goat anti-rabbit IgG (Dako, Glostrup, Denmark; diluted 1:400), rabbit anti-rat IgG (Sigma-Aldrich, diluted 1:40; Dako, diluted 1:100) monoclonal anti-guinea pig IgG (SigmaAldrich; diluted 1:40) were applied for 30 minutes. For staining of PlGF and VEGF, 1 drop of 3-amino-9ethylcarbazole (AEC+; Dako) was added, and sections were counterstained for 1 minute in hematoxylin. For immunofluorescence staining, after three washes in PBS for 10 minutes each and a final rinse in $10 \mathrm{mM}$ Tris$\mathrm{HCl}$ buffer (pH 8.8), labeling was analyzed under an inverted microscope equipped with epifluorescence optics. Specificity of staining (not shown) was assessed either by substitution of nonimmune serum for primary antibody, or for VEGF, by incubation with excess of blocking peptide (sc-152P; Santa Cruz Biotechnology).

\section{Statistical Analysis}

Data were analyzed for statistical significance $(P<0.05)$ on computer (Prism 3.0; GraphPad, San Diego, CA). The Mann-Whitney, Wilcoxon, and $\chi^{2}$ test (with Yates' correction) were used.

\section{RESULTS}

\section{VEGF Family Expression Profile in Human Neovascular Membranes}

VEGF-A, -B, and -C; PlGF; VEGFR-1 and -2 mRNA were detected in all CNV specimens obtained during surgery (Fig. 1). VEGF-D and VEGFR-3 were detected inconsistently (not shown). These neovascular membranes shared in common an aggressive history and probably represent a late stage of macular disease. Therefore, to evaluate more precisely the early spatial and temporal expression profiles of VEGF-A isoforms and of PlGF, RT-PCR analysis was applied on laser-induced murine neovascular choroidal membranes at different end points after laser treatment.

\section{RT-PCR Study of VEGF Isoforms}

VEGF protein was immunohistochemically detected in the intact neural retina, in the choroid, and in the neovascular membrane as previously described ${ }^{29}$ (Fig. 2A). We applied LPC technology to evaluate the pattern of VEGF-A expression specifically in the CNV compared with adjacent intact neural retina. The expression of the three murine VEGF-A isoforms mRNAs $\left(\mathrm{VEGF}_{120}, \mathrm{VEGF}_{164}\right.$, and $\left.\mathrm{VEGF}_{188}\right)$ was evident in the intact neural retina and choroid (Fig. 2B). The expression of the VEGF $_{120}$ isoform mRNA was predominant in early stages of $\mathrm{CNV}$ induced by laser treatment. Because the specific expression of VEGF ${ }_{120}$ mRNA had not been previously associated with the induction of murine endothelial cell proliferation, we performed an RT-PCR analysis in another unrelated murine model of angiogenesis, the aortic ring model. That model enables the separation of the parental aortic segment from the endothelial cells (Fig. 2D, EC) dividing and migrating in the collagen gel (Fig. 2C). VEGF $_{120}$ mRNA was also preferentially expressed in proliferating endothelial cells migrating in the gel, whereas both $\mathrm{VEGF}_{120}$ and $\mathrm{VEGF}_{164}$ were expressed in the parental aortic rings (Fig. 2D).

\section{RT-PCR Study of PIGF}

Unlike VEGF-A, PIGF protein was not detected in murine neural retina. It colocalized with collagen type IV and appeared to be concentrated in the choroidal layer and at the leading edge of the CNV (Figs. 3A-D). To evaluate the spatial pattern of PlGF mRNA expression, we performed RT-PCR on intact neural retina, intact choroid, and CNV after LPC (Fig. 3E). This confirmed the observations obtained at the protein level. PlGF mRNA was expressed in the intact choroid, was almost undetectable in neural retina, and was expressed in the CNV. The apparent low expression of PlGF mRNA at day 3 after laser treatment could correspond to limited amounts of neovascular elements that can be extracted by LPC at that early stage. In intact human donor eyes, PlGF mRNA was also preferentially expressed in the choroidal layer (Fig. 3F). To evaluate semi-quantitatively the temporal change in PlGF mRNA expression (which is not reliable with the LPC method), RT-PCR analysis was performed on mouse ocular posterior segments subjected to a larger number of laser-induced ruptures of Bruch's membrane (Figs. 3G, 3H). This demonstrated a statistically significant upregulation of PlGF expression during initial CNV development at days 3 and 5, with a return to basal expression levels after day 10 (coinciding with the period of CNV stabilization). 
FIGURE 2: $V E G F_{120}$ is the main isoform expressed in early stages of murine $C N V(A, B)$ and in the aortic ring model $(C, D)$. (A) VEGF-A protein was immunostained both in the CNV and in the neural retina at day 14 after laser treatment. (B) LPC followed by RT-PCR analysis of VEGF-A isoforms in microdissected choroidal neovessels (A, dotted line) growing in the subretinal space (CNV), in adjacent intact retinal tissue (R) and in normal choroid (Ch) from days 3 to 14 . VEGF $_{120}$ isoform (275 bp) appeared to be primarily expressed in these early stages of murine CNV development. (C) Photomicrograph of collagen-embedded aortic ring expiant isolated from WT mice and cultured for 7 days in autologous serum. (D) RT-PCR analysis of parental rings (dotted line) and endothelial cells (EC) showed the predominant expression of $\mathrm{VEGF}_{120}$ isoform $(*)$ in proliferating endothelium, whereas the two major isoforms $\left(\mathrm{VEGF}_{120}\right.$ and VEGF $\left._{164}\right)$ were expressed in the parental rings. The position of the synthetic internal control RNA for VEGF is at $311 \mathrm{bp}, \mathrm{VEGF}_{120}$ at 275 bp, VEGF 164 at 407 bp, and VEGF 188 at 479 bp. M, molecular marker. Original magnification: (A, C) x 200.
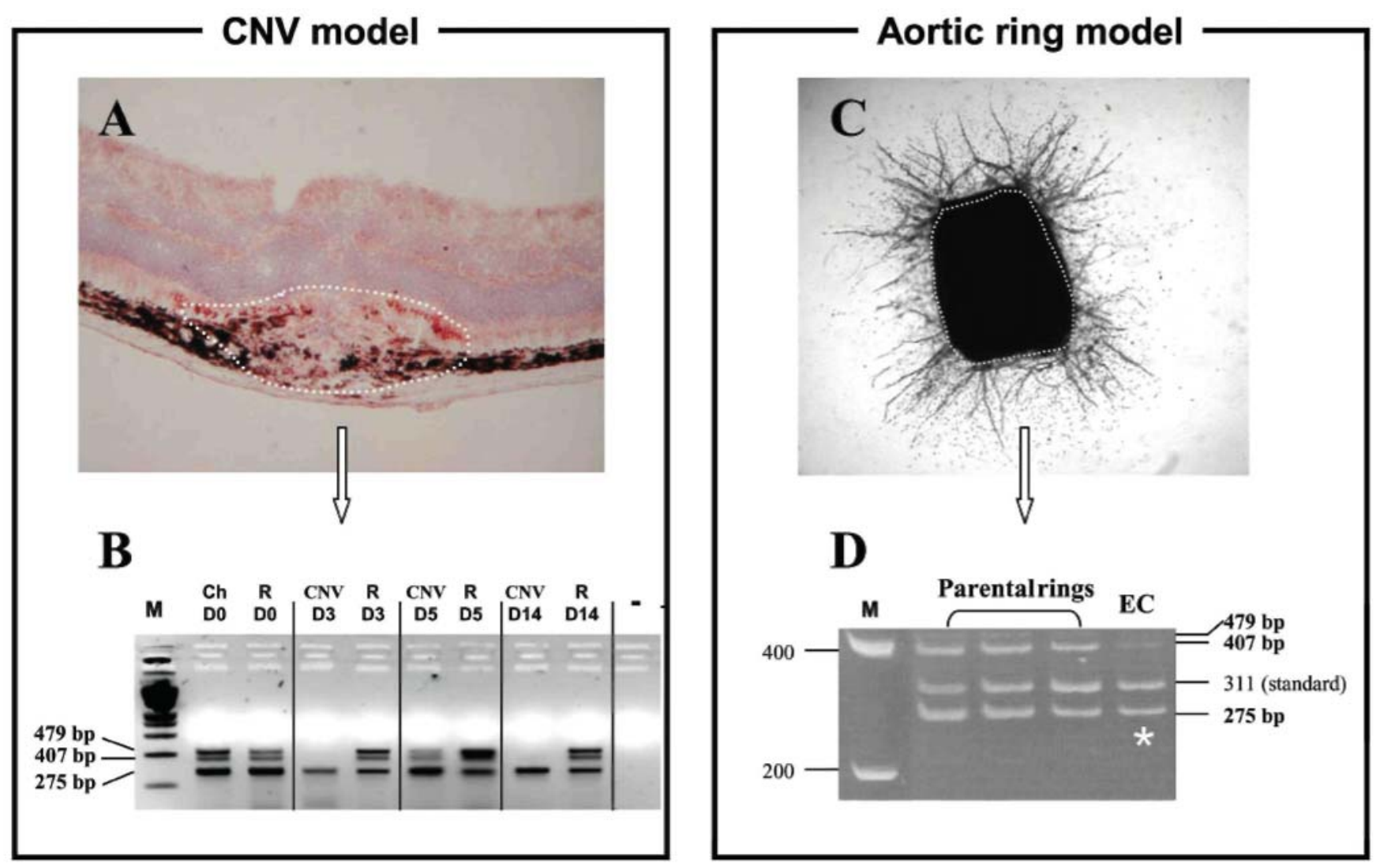

\section{CNV In Vivo}

To determine whether the absence of PlGF influenced CNV in vivo, we first evaluated the efficiency of CNV lesion development in PlGF deficient and WT mice. In $\mathrm{PlGF}^{-/}$mice, most of the lesions were detectable only indirectly through the trauma observed in the photoreceptor layer, whereas in $\mathrm{PlGF}^{+/+}$control specimens, CNV development with endothelial (identified by anti-PECAM immunostaining) and pigmented cells was clearly identified (Figs. 4A-D). Choroidal newly formed microvessels growing over the plane of RPE were present in $81.2 \%$ of the laser-induced lesions in $\mathrm{PlGF}^{+/+}$versus $18.5 \%$ in PlGF $^{-/-}$mice $\left(\chi_{\mathrm{Y}}^{2}=11.2, P<0.001\right)$. Severity of neovascularization was then estimated histologically, by measuring on serial sections the maximum height of the lesion above the choroidal layer observed in neighboring intact zones, as previously described. This was quantified by determining the B-C ratio between total thickness of lesions (B), from the bottom of the choroid to the top of the neovascular area to the thickness of adjacent normal choroid (C), and a 70\% reduction of the B-C ratio was consistently observed in PlGF-deficient mice $(P<0.001)$ compared with WT mice (Fig. 4E).

Compared with control mice treated with IgG, anti-Fltl-treated mice developed significantly $(P<0.001)$ smaller CNV. This was evidenced both by measuring CNV surface on choroidal flatmounts ( $60 \%$ reduction) and by calculating the B-C ratio (40\% reduction) on frozen sections (Fig. 5). 
FIGURE 3: Immunolocalization and mRNA expression of PlGF in laser-induced CNV. (A-C) Immunofluorescence labeling of PIGF (red) and colocalization with collagen type IV (green) at day 14 after laser-induced CNV. (D) PIGF protein was immunostained in the neovascular leading edge and in the choroidal (Ch) layer (yellow arrows) Ret, retina. (E) LPC followed by RT-PCR analysis of intact choroid (Ch), microdissected choroidal neovessels (dotted line) growing in the subretinal space (CNV), and adjacent intact neural retina (R) at day 3, 5, and 14. The spatial profile of PIGF mRNA paralleled the localization of the protein, with expression in the intact choroidal layer and in the CNV, and almost undetectable expression in neural retina. (F) PlGF mRNA was also present in human normal posterior segments, with a predominant expression in the RPE-choroid complex (Ch). (G) Representative gel of PIGF and 28S mRNA expression evaluated on the entire ocular posterior segment by RT-PCR at different end points (day 3-40) after laser-induced trauma in mice. (H) The histogram corresponds to the densitometric quantification of PlGF mRNA normalized to the 28S signal $(n=12)$. There was a statistically significant induction of PIGF mRNA at days 3 and 5 after laser treatment, compared with the level of PIGF mRNA expressed at day 0 . The positions of murine and human PIGF mRNA are at 130 and $164 \mathrm{bp}$, respectively. ${ }^{* *} P<0.01,{ }^{* * *} P<0.001$; error bars, SE; M, molecular marker. Original magnification: (A-C) x400; (D) x200.
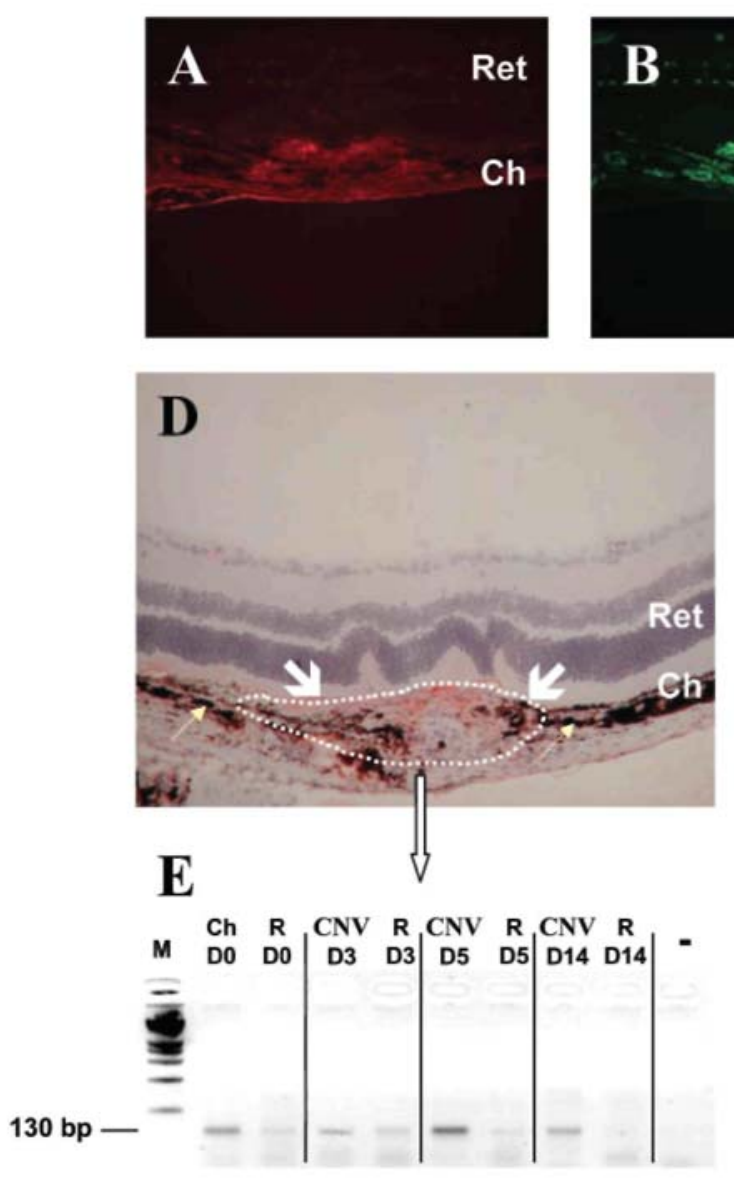

R Ch R Ch R Ch - M

$\mathbf{F}$
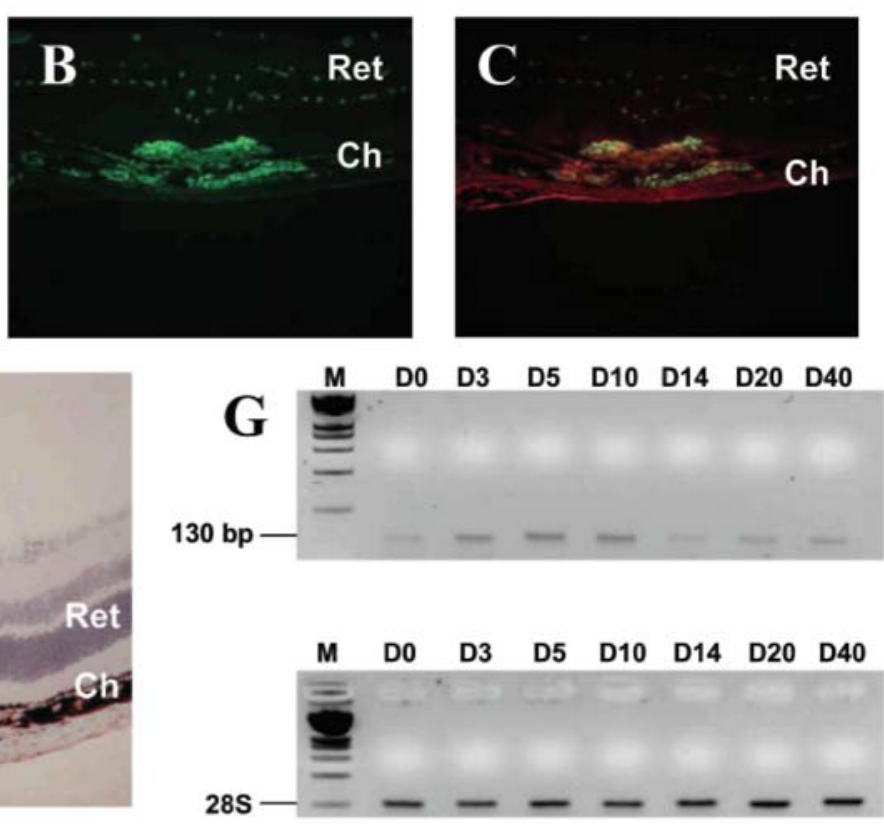

$\mathbf{H}$

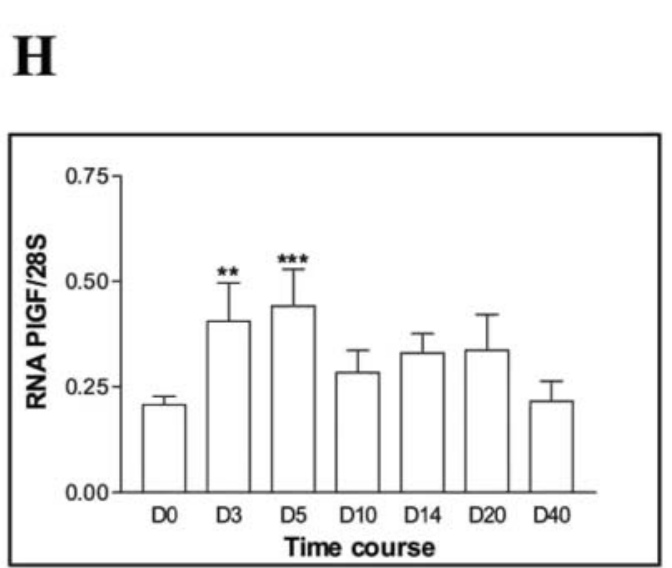
Time course

\section{$\equiv$}

$164 \mathrm{bp}-\cdots-\cdots$

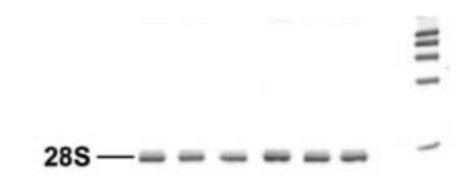


FIGURE 4: Absence of PlGF prevented the development of CNV. (A) Hematoxylin-eosin staining of a representative area of $\mathrm{CNV}$ at the site of laser-induced trauma and (B) immunofluorescence labeling of neovessels in PlGF ${ }^{+/+}$mice. New vessels were detected with colocalization of anti-mouse anti-collagen type IV antibody (red) and anti-mouse anti-PECAM antibody (green). (C, D) Almost complete absence of neovascularization was visible in PIGF $^{-1-}$ mice both in terms of neovascular volume and lateral extension. (E) The neovascular reaction was determined with computer-assisted image analysis by evaluating the B-C ratio, as previously described ${ }^{23,24}$ at day 14 after laser injury of the Bruch's membrane in WT mice and in the limited number of laser impact sites developing a measurable reaction in PlGFdeficient mice. The neural retina (Ret) and choroidal layer $(\mathrm{Ch})$ are indicated. Arrows: the neovascular area. Original magnification: (A, C) x200: (B, D) x400; error bars, SE.
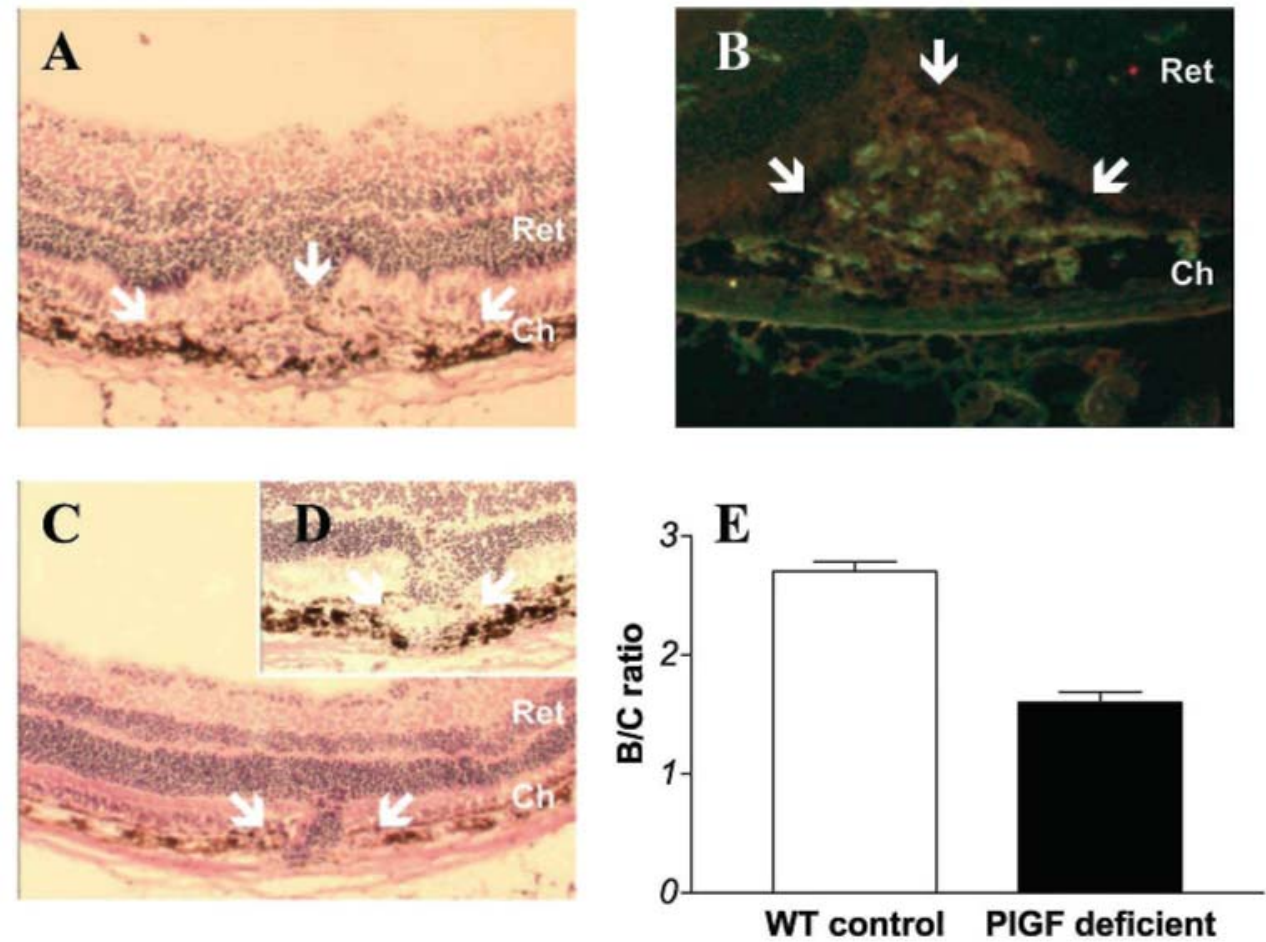

\section{DisCUSSION}

We report here that, in addition to VEGF-A, other members of the VEGF family and their receptors are also expressed in late stages of human exudative AMD. This study completes a previous report demonstrating, by immunohistochemistry, the presence of VEGF-B and -C, and PIGF proteins in human choroidal neovascular membranes. ${ }^{17}$ PlGF mRNA was also expressed in intact murine and human choroidal layer. The redundancy in expression of angiogenic factors has been associated with aggressive vascular remodeling and/or tumor progression. ${ }^{30}$ Our observations suggest that, as in other tissues subjected to pathologic angiogenesis, CNV formation is the result of a complex balance of pro- and antiangiogenic influences not limited to isolated overexpression of VEGF-A. Although the three VEGF-A major isoforms were expressed at a basal rate in intact murine choroidal layer and neural retina, $\mathrm{VEGF}_{120}$ mRNA was the isoform primarily induced in early stages of experimental murine CNV (extracted by LPC) and, in vitro, in proliferating endothelial cells migrating from an aortic ring segment. It is obviously difficult to evaluate the relevance of these results to the situation in the human eye. The three VEGF-A isoforms were clearly expressed in human neovascular membranes extracted at a late stage, during macular translocation. Furthermore, the murine model of CNV represents merely a woundhealing response, and the aortic ring model is an in vitro assay unrelated to ocular neovascularization. Similar observations in two completely different models make attractive the alternative hypothesis that a specific VEGFA isoform (not necessarily $\mathrm{VEGF}_{120}$ ) may be selectively expressed during the initiation of human CNV.

Complete data are lacking to appreciate definitively the relative importance of each member of the VEGF family in the development of exudative AMD. Animals with systemic disruption of candidate genes are lethal, and mice with inducible silencing of the VEGF-A gene in the posterior segment have not yet been produced. The importance of VEGF-A has been recognized based on the demonstration that VEGF mRNA and protein were present in pathologic specimens, ${ }^{3}$ induced by hypoxia in ischemic retinopathies ${ }^{31,32}$ and on the observations that therapeutic strategies aiming at inhibiting VEGF-A (through repeated injections of a blocking antibody or through blockade of its receptor) reduced the development of CNV ${ }^{6,7}$ However, experimentally, blockade of a single player in the complex spectrum of angiogenic actors is usually sufficient to significantly inhibit pathologic 
neovascularization. ${ }^{33}$ Furthermore, although VEGF expression is probably necessary, isolated overexpression of VEGF is not sufficient for the development of CNV growing under the retina, suggesting the involvement of other proangiogenic mechanisms. ${ }^{10,11}$ We investigated therefore the influence of PlGF on the development of experimental CNV, because (1) PlGF expression was present both in human and experimental CNV (this study) and (2) PlGF-deficient mice, contrary to VEGF-deficient animals, which do not survive, develop normally and demonstrate an abnormal phenotype in various pathologic conditions. ${ }^{18}$

PlGF deficiency or PlGF receptor neutralization both induced a very significant reduction in the incidence and in the severity of laser-induced CNV compared with control animals.

FIGURE 5: Inhibition of $C N V$ with anti-Fltl antibody. After induction of $C N V$, WT mice were injected three times a week with anti-Fltl antibody $(C, D)$ or control $\operatorname{Ig} G(A, B)$. Mice treated with anti-Fltl showed CNV lesions that were smaller than those in controls both on choroidal flat-mounts examined by fluorescence microscopy (A, C) and on frozen sections counterstained with hematoxylin-eosin (B, D). This was quantified by measuring CNV surface (E) and calculating the B-C ratio (F), and a statistically significant reduction $(P<0.001)$ in CNV formation was observed in WT mice treated with anti-Fltl compared with the control. Error bars, SE. Original magnification, $\mathrm{x} 200$.
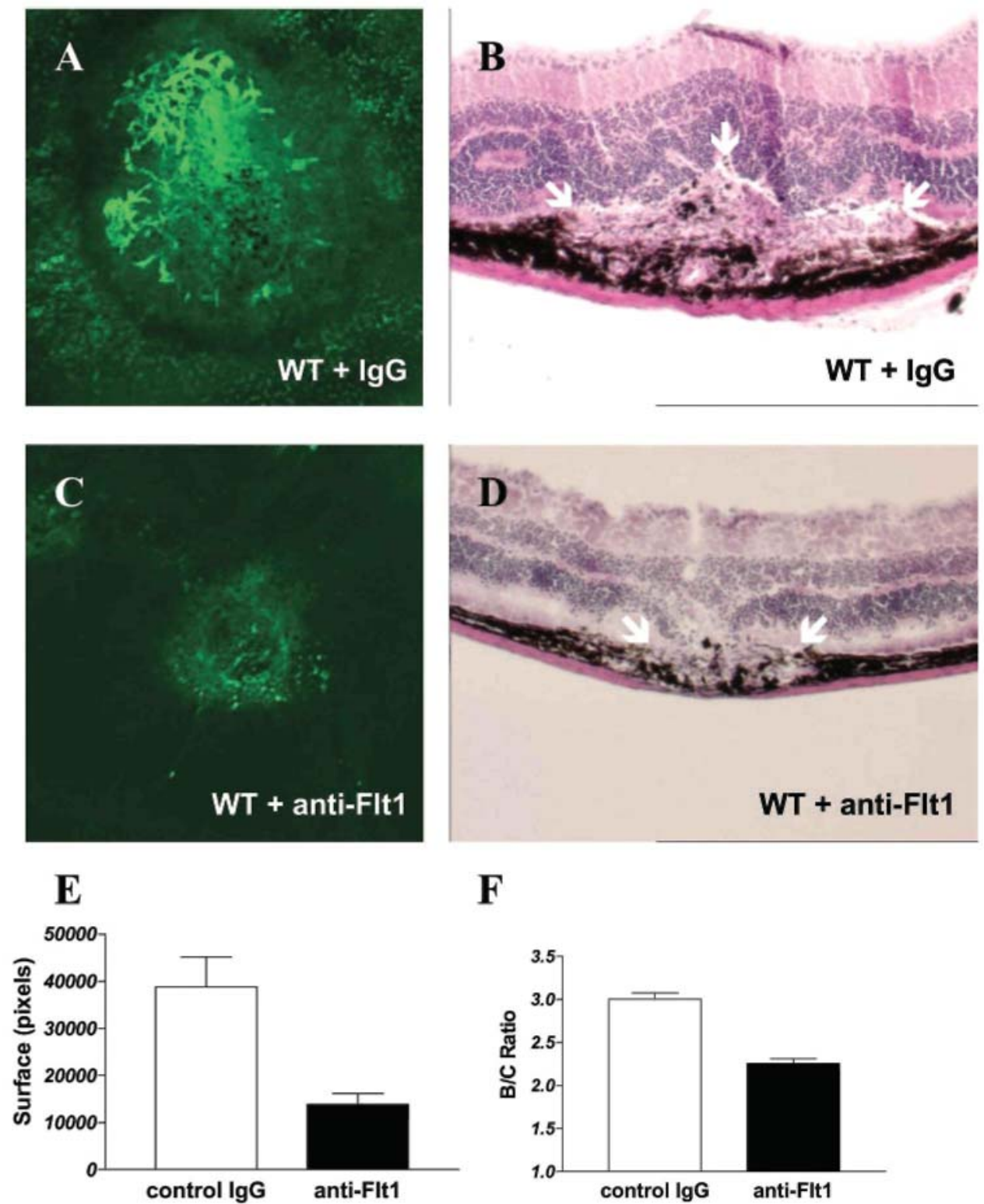

F

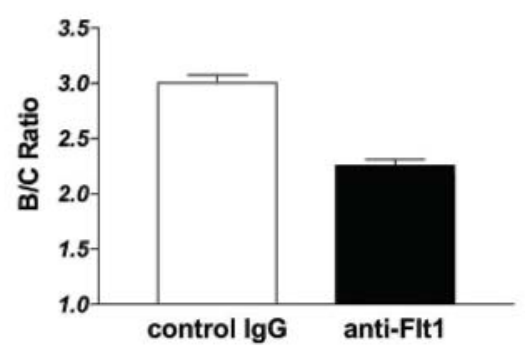


The resistance of PIGF-deficient mice to pathologic angiogenesis is in accordance with previously published results in hypoxic retina, in an aortic ring model and in cancer. ${ }^{18}$ PlGF could enhance angiogenesis on its own through activation of VEGFR-1, ${ }^{19}$ by forming heterodimers with VEGF-A, ${ }^{34}$ or by displacing VEGF-A from VEGFR-1. ${ }^{35}$ PlGF could also mobilize bone-marrow- derived cells and inflammatory cells. ${ }^{18,25}$ The influence of inflammatory components in AMD and in experimental CNV has recently been pointed out by different groups. ${ }^{23,36,37}$ Furthermore, circulating angioblasts were recently shown to contribute to ischemic retinal neovascularization. ${ }^{38}$ The precise mechanisms in the eye obviously need further evaluation. Nevertheless, together with the pattern of PlGF mRNA expression obtained in experimental CNV and in surgically extracted neovascular membranes, our observations in PIGF-deficient mice suggest a participation of PlGF and its receptor Flt1 in the development of exudative AMD.

The efficacy of VEGF blockade in angiogenesis inhibition is not in dispute, and there is no doubt that VEGF and/or its receptors are currently a promising potential target for antiangiogenesis therapy. Our neutralization experiment with anti-Flt1 antibody blocked the interactions of PlGF but also of other Flt1 ligands, such as VEGF-A or -B. ${ }^{16}$ In contrast, a recent study demonstrated in a tumoral model that combined inhibition of VEGFR-1 and -2 was more efficient than a single approach in inhibiting VEGF effects. ${ }^{39}$ Moreover, a recent study demonstrated that VEGF had neuroprotective and neuroregenerative effects on the retina. ${ }^{40}$ Our observations could therefore be of importance not only for a better understanding of CNV physiopathology but also for the treatment of the exudative form of AMD, especially in view of the unavailability of any data concerning the safety of long-term VEGF absence on retinal biology.

\section{Acknowledgments}

The authors thank Laurent Naa and Fabrice Olivier for their collaboration and Patricia Gavitelli for technical assistance.

Disclosure: J.-M. Rakic, None; V. Lambert, None; L. Devy, None; A. Luttun, None; P. Carmeliet, None; C. Claes, None; L. Nguyen, None; J.-M. Foidart, None; A. Noe“l, None; C. Munaut, None

\section{References}

1. Miller JW, Adamis AP, Aiello LP. Vascular endothelial growth factor in ocular neovascularization and proliferative diabetic retinopathy. Diabetes Metab Rev. 1997;13:37-50.

2. Campochiaro PA. Retinal and choroidal neovascularization. J Cell Physiol 2000;184:301-310.

3. Kvanta A, Algvere PV, Berglin L, Seregard S. Subfoveal fibrovascu-lar membranes in age-related macular degeneration express vascular endothelial growth factor. Invest Ophthalmol Vis Sci. 1996;37: 1929-1934.

4. Yi X, Ogata N, Komada M, et al. Vascular endothelial growth factor expression in choroidal neovascularization in rats. Graefes Arch Clin Exp Ophthalmol. 1997;235:313-319.

5. Wada M, Ogata N, Otsuji T, Uyama M. Expression of vascular endothelial growth factor and its receptor (KDR/flk-1) mRNA in experimental choroidal neovascularization. Curr Eye Res. 1999;18: 203-213.

6. Krzystolik MG, Afshari MA, Adamis AP, et al. Prevention of experimental choroidal neovascularization with intravitreal anti-vascular endothelial growth factor antibody fragment. Arch Ophthalmol. 2002;120:338-346.

7. The Eyetech Study Group. Preclinical and phase 1A clinical evaluation of an anti-VEGF pegylated aptamer (EYE001) for the treatment of exudative age-related macular degeneration. Retina. 2002; 22:143-152.

8. Gilbert RE, Vranes D, Berka JL, et al. Vascular endothelial growth factor and its receptors in control and diabetic rat eyes. Lab Invest. 1998;78:1017-1027.

9. Iruela-Arispe ML, Dvorak HF. Angiogenesis: a dynamic balance of stimulators and inhibitors. Thromb Haemost. 1997;78:672-677.

10. Okamoto N, Tobe T, Hackett SF, et al. Transgenic mice with increased expression of vascular endothelial growth factor in the retina: a new model of intraretinal and subretinal neovascularization. Am J Pathol. 1997;151:281-291.

11. Schwesinger C, Yee C, Rohan RM, et al. Intrachoroidal neovascularization in transgenic mice overexpressing vascular endothelial growth factor in the retinal pigment epithelium. Am J Pathol. 2001;158:1161-1172. 
12. Soubrane G, Cohen SY, Delayre T, et al. Basic fibroblast growth factor experimentally induced choroidal angiogenesis in the minipig. Curr Eye Res. 1994;13:183-195.

13. Spilsbury K, Garrett KL, Shen WY, Constable IJ, Rakoczy PE. Overexpression of vascular endothelial growth factor (VEGF) in the retinal pigment epithelium leads to the development of choroidal neovascularization. Am J Pathol. 2000;157:135-144.

14. Ohno-Matsui K, Morita I, Tombran-Tink J, et al. Novel mechanism for age-related macular degeneration: an equilibrium shift between the angiogenesis factors VEGF and PEDF. J Cell Physiol. 2001;189: 323-333.

15. Renno RZ, Youssri AI, Michaud N, Gragoudas ES, Miller JW. Expression of pigment epithelium-derived factor in experimental choroidal neovascularization. Invest Ophthalmol Vis Sci. 2002;43: 1574-1580.

16. Neufeld G, Cohen T, Gengrinovitch S, Poltorak Z. Vascular endothelial growth factor (VEGF) and its receptors. FASEB J. 1999; 13: 9-

17. Otani A, Takagi $\mathrm{H}, \mathrm{Oh} \mathrm{H}$, et al. Vascular endothelial growth factor family and receptor expression in human choroidal neovascular membranes. Microvasc Res. 2002;64:162-169.

18. Carmeliet P, Moons L, Luttun A, et al. Synergism between vascular endothelial growth factor and placental growth factor contributes to angiogenesis and plasma extravasation in pathological conditions. Nat Med. 2001;7:575-583.

19. Ziche M, Maglione D, Ribatti D, et al. Placenta growth factor-1 is chemotactic, mitogenic and angiogenic. Lab Invest. 1997;76:517-531.

20. Simpson DA, Murphy GM, Bhaduri T, Gardiner TA, Archer DB, Stitt AW. Expression of the VEGF gene family during retinal vasoobliteration and hypoxia. Biochem Biophys Res Commun. 1999; 262:333-340.

21. Castellon R, Hamdi HK, Sacerio I, et al. Effects of angiogenic growth factor combinations on retinal endothelial cells. Exp Eye Res. 2002;74:523-535.

22. Khaliq A, Foreman D, Ahmed A, et al. Increased expression of placenta growth factor in proliferative diabetic retinopathy. Lab Invest. 1998;78:109-116.

23. Lambert V, Munaut C, Jost M, et al. Matrix metalloproteinase-9 contributes to choroidal neovascularization. Am J Pathol. 2002; 161:1247-1253.

24. Lambert V, Munaut C, Noel A, et al. Influence of plasminogen activator inhibitor type I on choroidal neovascularization. $F A S E B J$. 2001;15:1021-1027.

25. Luttun A, Tjwa M, Moons L, et al. Revascularization of ischemic tissues by PlGF treatment, and inhibition of tumor angiogenesis, arthritis and atherosclerosis by anti-Flt1. Nat Med. 2002;8:831-840.

26. Edelman JL, Castro MR. Quantitative image analysis of laser-induced choroidal neovascularization in rat. Exp Eye Res. 2000;71: 523533.

27. Mori K, Ando A, Gehlbach P, et al. Inhibition of choroidal neovascularization by intravenous injection of adenoviral vectors expressing secretable endostatin. Am J Pathol. 2001;159:313-320.

28. Devy L, Blacher S, Grignet-Debrus C, et al. The pro- or antiangio-genic effect of plasminogen activator inhibitor 1 is dose dependent. FASEB J. 2002;16:147-154.

29. Kim I, Ryan AM, Rohan R, et al. Constitutive expression of VEGF, VEGFR-1 and VEGFR-2 in normal eyes. Invest Ophthalmol Vis Sci. 1999;40:2115-2121.

30. Eggert A, Ikegaki N, Kwiatkowski J, Zhao H, Brodeur GM, Himel-stein BP. High-level expression of angiogenic factors is associated with advanced tumor stage in human neuroblastomas. Clin Cancer Res. 2000;5:1900-1908.

31. Pierce EA, Avery RL, Foley ED, Aiello LP, Smith LE. Vascular endothelial growth factor/vascular permeability factor expression in a mouse model of retinal neovascularization. Proc Natl Acad Sci USA. 1995;92:905-909.

32. Pe'er J, Shweiki D, Itin A, Hemo I, Gnessin H, Keshet E. Hypoxia-induced expression of vascular endothelial growth factor by retinal cells is a common factor in neovascularizing ocular diseases. Lab Invest. 1995;72:638-645.

33. Yancopoulos GD, Davis S, Gale NW, Rudge JS, Wiegand SJ, Holash J. Vascular-specific growth factors and blood vessel formation. Nature. 2000;407:242-248.

34. Cao Y, Chen H, Zhou L, et al. Heterodimers of placenta growth factor/vascular endothelial growth factor: endothelial activity, tumor cell expression, and high affinity binding to Flk-1/KDR. J Biol Chem. 1996;271:3154-3162.

35. Park JE, Chen HH, Winer J, Houck KA, Ferrara N. Placenta growth factor, potentiation of vascular endothelial growth factor bioactiv- 
Published in: Investigative Ophthalmology \& Visual Science (2003), vol.44, iss.7, pp. 3186-3193

Status: Postprint (Author's version)

ity, in vitro and in vivo, and high affinity binding to Flt-1 but not to Flk-1/KDR. J Biol Chem. 1994;269:25646-25654.

36. Penfold PL, Madigan MC, Gillies MC, Provis JM. Immunological and aetiological aspects of macular degeneration. Prog Retinal Eye Res. 2001;20:385-414.

37. Hageman GS, Luthert PJ, Victor Chong NH, Johnson LV, Anderson DH, Mullins RF. An integrated hypothesis that considers drusen as biomarkers of immune-mediated processes at the RPE-Bruch's membrane interface in aging and age-related macular degeneration. Prog Retinal Eye Res. 2001;20:705-732.

38. Grant MB, May WS, Caballero S, et al. Adult hematopoietic stem cells provide functional hemangioblast activity during retinal neovascularization. Nat Med. 2002;8:607-612.

39- Lu D, Jimenez X, Zhang H, et al. Complete inhibition of vascular endothelial growth factor (VEGF) activities with a bifunctional diabody directed against both VEGF kinase receptors, fms-like tyrosine kinase receptor and kinase insert domain-containing receptor. Cancer Res. 2001;61:7002-7008.

40. Böcker-Meffert S, Rosenstiel P, Röhl C, et al. Erythropoietin and VEGF promote neural outgrowth from retinal expiants in postnatal rats. Invest Ophthalmol Vis Sci. 2002;43:2021-2026. 